\title{
An Unusual Presentation of Alveolar Type of Rhabdomyosarcoma of Orbit
}

\author{
Bastola P, ${ }^{1}$ Adhikari RK ${ }^{1}$ \\ 'Lumbini Eye Institute, Shree Rana Ambika Shah Eye Hospital, Bhairahawa, Rupendehi Nepal.
}

\section{ABSTRACT}

Orbital rhabdomyosarcoma is the commonest primary orbital malignancy in children. A case of alveolar type of orbital rhabdomyosarcoma with unusual presentation in a three and half years male child presented with painless nodular swelling in the left lower lid of one and half years duration and gradual protrusion of the left eyeball. Examination of the patient revealed a mass in the left lower lid region extending to other parts of orbit and lymphnode metastasis in the neck region along with non axial proptosis, rest of the systemic examination being normal. Patient underwent incisional biopsy of the mass and excisional biopsy of the lymphnodes which revealed alveolar type of primary orbital rhabdomyosarcoma with lymphnode metastasis.

Key Words: alveolar rhabdomyosarcoma, lymphnode metastasis, orbit, proptosis

\section{INTRODUCTION}

Rhabdomyosarcoma (RMS) is the most common soft tissue sarcoma in children. Weber first described rhabdomyosarcoma in 1854; histological definition was available in 1946, when Stout recognized the distinct morphology of rhabdomyoblasts. Stout described rhabdomyoblasts as appearing in round, strap, racquet, and spider forms. ${ }^{1}$ Approximately $15 \%$ of all rhabdomyosarcomas occur in the orbit. ${ }^{2}$ Rhabdomyosarcoma infiltrate and metastasize through blood and lymphatic channels. Sites for metastasis are lung, bone and liver, spread to lymphnodes is in only $10 \%$ of cases. Orbital Rhabdomyosarcoma was first reported by Bayer. ${ }^{3}$

\section{CASE REPORT}

A three and half years old male child presented with a nodular mass in the left lower lid along with protrusion of the left eyeball for one and half years. He gave history of seeking treatment at various hospital but symptoms got worse with increasing pain, redness of the eye, diminution of vision along with marked protrusion of the left eyeball. Ocular examination of the child revealed a visual acuity of "following and fixating the light" with both the eyes. An examination finding of the Right eye was normal. In the left eye there was diffuse eyelid swelling with tortuous vessels over it, hyperemic lid skin, with lagophthalmos (Figure 1). Multiple non tender, rubbery lymph nodes measuring around 0.5 to $2 \mathrm{~cm}$ in

Correspondence:

Dr. Pradeep Bastola

Lumbini Eye Institute

Shree Rana Ambika Shah Eye Hospital

Siddharthanagar-3, Bhairahawa, Rupendehi,

Nepal.

Email: pbs_dinku@yahoo.com

Phone: 977-9841444177 
size were present in submandibular region, few were matted, local temperature of the lymph nodes was not raised, and lymph nodes were not attached with overlying skin but were immobile. Non-axial protrusion of the left eyeball was present. Globe was displaced superotemporally. A firm tender mass of around $6 \times 6.5$ $\mathrm{cm}$, was located in the inferior part of globe, which was extending posteriorly, base was not palpable, the mass was immobile, it was not separated from the globe, there was no bruit heard on auscultation over the mass. Pulsation was absent and there was no retropulsion of the mass as well. Crust with Keritinization of bulbar as well as lower tarsal conjunctiva was present, congestion and chemosis of the conjunctiva was also present. There were periorbital changes in the left eye. Rest of the examination findings regarding the left eye ball was difficult to assess, however Red reflex was present in distance direct ophthalmoscopy.

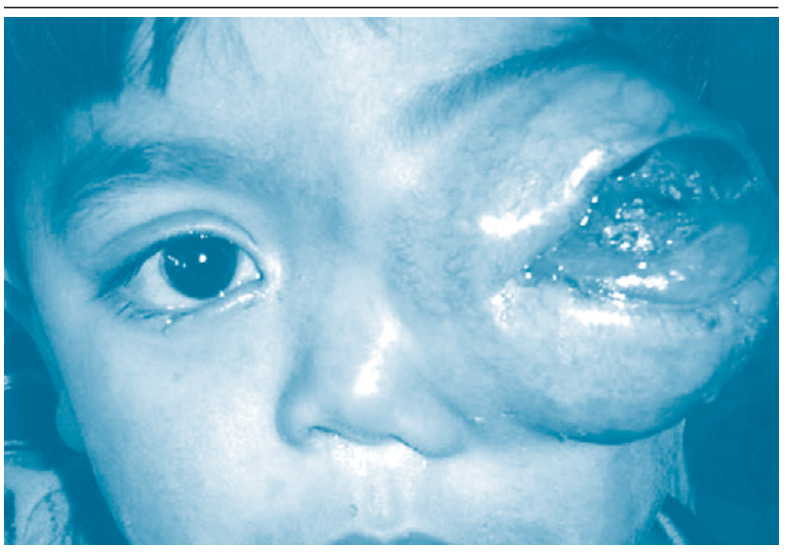

Figure 1. The status of the child at initial presentation

After a detailed ocular examination a provisional diagnosis was made of left orbital rhabdomyosarcoma with a differential diagnosis of left retinoblastoma with orbital extension in mind. The patient was advised Computed tomography (CT) scan of head and orbit which showed large ill-defined predominantly homogenous soft tissue attenuation mass lesion of approximately $55 \times 52 \mathrm{~mm}$ size, involving left orbit projecting anteriorly and showing extension into the retro orbital region with obscuration of whole of the orbital and retro orbital structures including optic nerve. However no obvious calcification or intracranial extension was noted (Figure 2). Routine blood investigations, urine examination were with in normal limits. Chest $\mathrm{X}$-ray along with ultrasound abdomen was advised, the reports showed no features of tumor extension (Figure 3). An incisional biopsy of the main tumor mass and excisional biopsy of enlarged lymph nodes was done. A thin longitudinal strip of mass from lower lid was taken during incisional biopsy. Similarly excisional biopsy of submandibular lymph node was taken in the same sitting. The report of histopathological examination showed proliferation of tumor cells arranged in pseudopapillae, alveolar pattern, lobules, and solid nests with multiple pseudo rosettes having central fibrovascualr core. It was composed of small round to oval cells, with minimal pleomorphism having scanty cytoplasm and hyperchromatic nuclei with inconspicuous nucleoli, some of the areas showed cells arranged in solid sheets. The section from cervical lymphnode showed almost complete effacement of nodal architecture replaced by tumor cells proliferation was similar to that seen in orbital mass (Figure 4). With all these histological features a pathological diagnosis of alveolar type of orbital rhabdomyosarcoma was made. Further investigations like liver function tests, renal function tests, bone marrow aspiration, serum electrolytes were done, all of the investigations came with in normal limits.

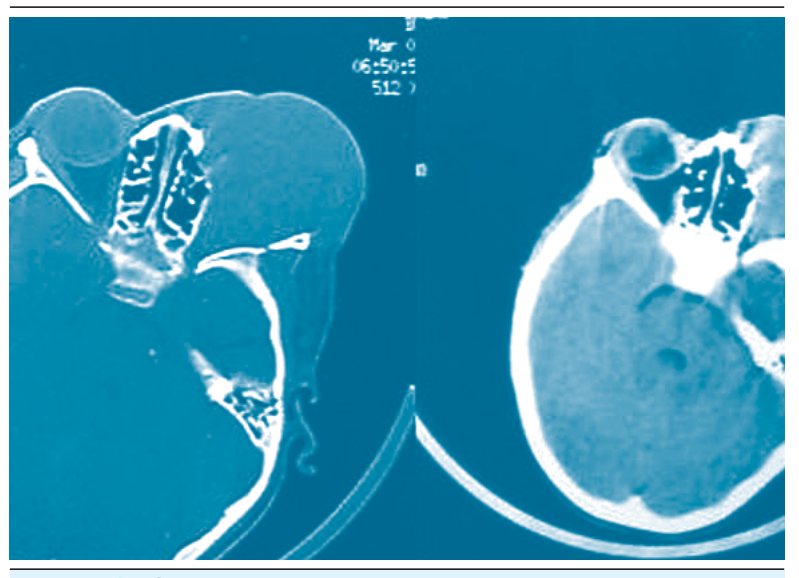

Figure 2. Computed tomography scan of head and orbit

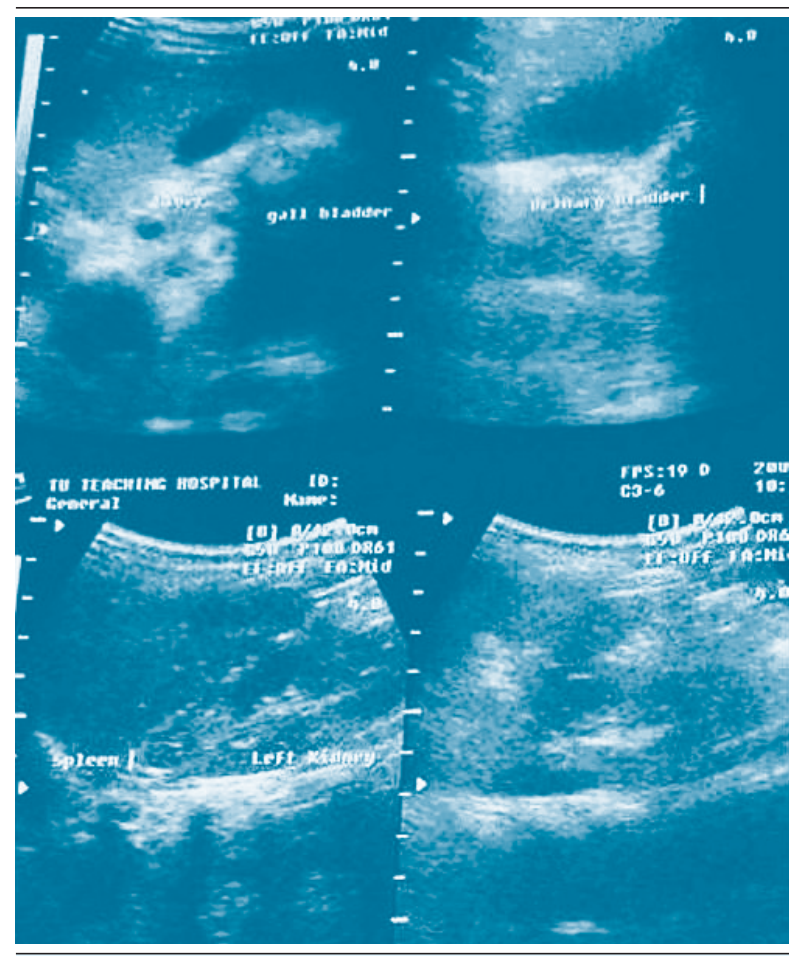

Figure 3. Normal ultrasound abdomen findings

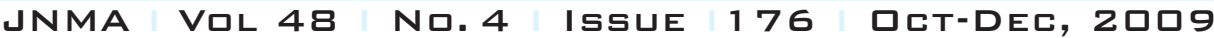




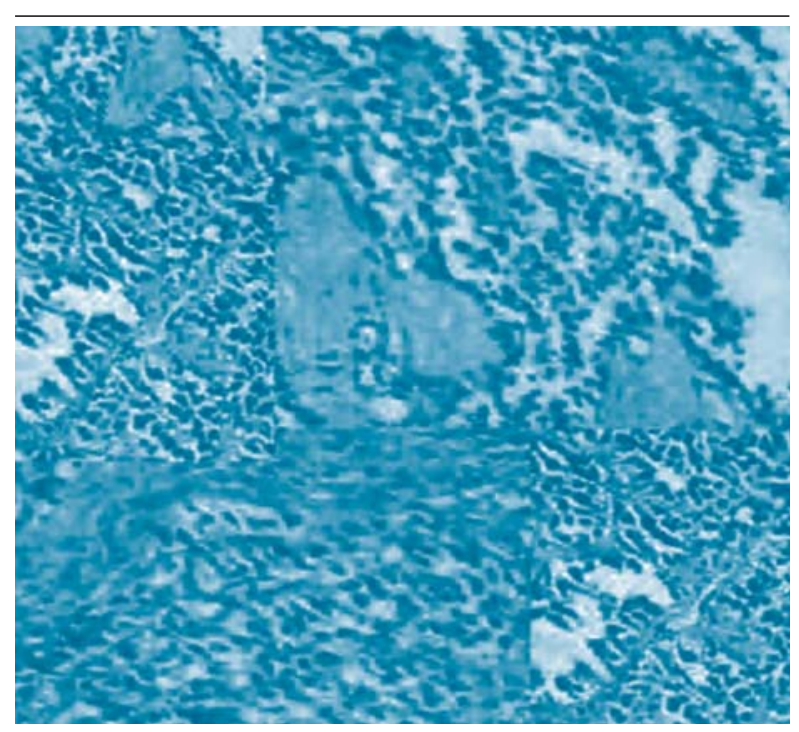

Figure 4. Histological features of the incised tumor mass and excised lymph nodes

Patient was transferred to paediatric oncology unit for chemoreduction and further planned for radiotherapy. Chemoinduction was done; the patient was followed up every day from ophthalmic side. As there was not much of improvement with chemotherapy, the pediatric oncologist advised for a surgical intervention. Patient did not come back to ophthalmology department; further inquiry revealed patient was discharged against medical advice.

\section{DISCUSSION}

Approximately $50 \%$ of soft tissue sarcomas in children are rhabdomyosarcomas. Rhabdomyosarcomas may occur anywhere in the body. Five subtypes of rhabdomyosarcoma have been described; however, most fall into one or two major subtypes, embryonal and alveolar. Other subtypes include botryoid, spindle cell, and undifferentiated. ${ }^{4}$ Formal investigations of the treatment of pediatric rhabdomyosarcoma have subsequently been carried out through the Intergroup Rhabdomyosarcoma Study (IRS) Group and have consisted of IRS-I from 1972-1978, IRS-II from 1978-1984, IRSIII from 1984-1991, IRS-IV from 1991-1997, and IRS V from 1997-2003. Current investigations are underway with IRS-VI. Treatment, once purely surgical, is now multimodal with conservative surgical resection, biopsy only, or surgical staging. Embryonal variant occurs most commonly and usually is found in the head and neck, genitourinary tract, or orbit. This subtype is characterized by a loss of heterozygosity at the $11 \mathrm{p} 15.5$ locus, the region of the IGFII gene. ${ }^{4}$

According to Rubin, rhabdomyosarcoma is derived from primitive mesenchyme that retained its capacity for skeletal muscle differentiation. Rhabdomyosarcoma of the head and neck is primarily a disease of the first decade of life, and it is the most common soft tissue sarcoma in childhood. Approximately $90 \%$ of all cases of rhabdomyosarcoma are diagnosed in individuals younger than 25 years, and within this group, 60-70\% are younger than 10 years. Rhabdomyosarcoma represents $3.5 \%$ of all malignancies in children aged 0-14 years, with approximately 250 new cases diagnosed each year. The annual incidence of rhabdomyosarcoma in the United States is 4.5 cases per one million children. ${ }^{5}$ The alveolar variant has a translocation involving the PAX3 locus, between the long arm of chromosome 2 and the long arm of chromosome 13. This involves the PAX3 gene and the FKHR gene. Alveolar rhabdomyosarcoma usually is encountered in the extremities, trunk, or perineum. PAX3-FKHR and PAX7-FKHR gene fusions have recently been shown to have a prognostic implication in patients with metastatic disease. It appears that, among gene fusion positive patients with the alveolar variant, the PAX3-FKHR gene fusion is an independent adverse prognosticator while the PAX7-FKHR gene fusion correlates with an improved prognosis. Gene fusion negative cases of alveolar histology appear to have an intermediate outcome in comparison to the gene fusion groups. $^{4}$

The botryoid variant, named because of its gross resemblance to a cluster of grapes, arises in cavitary structures such as the vagina and bladder and is found more frequently in infants. Spindle cell rhabdomyosarcoma, found most commonly in the paratesticular area, is another variant of the embryonal histology. ${ }^{4}$ The alveolar rhabdomyosarcoma differs from the embryonal type in many respects. The embryonal type has a predilection for the superior nasal quadrant, while the alveolar type is seen more often in the lower part of the orbit. ${ }^{5}$ The growth in our patient also appeared for the first time in the inferior part of the orbit. The alveolar type of rhabdomyosarcoma is believed to be a highly malignant tumour. Secondaries due to this tumour have been described in brain, paranasal sinuses and lungs. Its extension in to the cervical lymph nodes has been described very rarely. ${ }^{6}$ In our patient, secondaries in the submandibular lymph nodes were present which was quite unusual and rare. The early involvement of the submandibular lymph nodes suggests its rapid lymphatic spread. In the similar manner in our case the progression of the disease pathology was very gradual and slow, however alveolar type of rhabdomyosarcoma has a more malignant course than embroynal or botryoid variants. The cause of RMS is unclear. ${ }^{1}$ Several genetic syndromes and environmental factors are associated with increased prevalence of RMS. Genetic syndromes include the following: Neurofibromatosis $(4-5 \%$ risk of any of a number of malignancies), Li-Fraumeni syndrome (germline

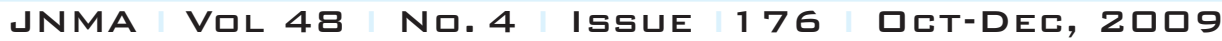


mutation of the tumor suppressor gene TP53), RubinsteinTaybi syndrome, Gorlin basal cell nevus syndrome, Beckwith-Wiedemann syndrome, Costello syndrome. Environmental factors appear to influence the development of RMS, as follows: Parental use of marijuana and cocaine, intrauterine exposure to X-rays, previous exposure to alkylating agents. ${ }^{1}$

\section{ACKNOWLEDGMENTS}

We would like to acknowledge paediatric oncology unit, Kanti Children Hospital, Kathmandu along with the department of pathology Tribhuvan University, Teaching Hospital, Maharajganj, Kathmandu and patient and the informant and B.P. Koirala Lions Centre for Ophthalmic Studies family.

\section{REFERENCES}

1. Timothy PC. Rhabdomyosarcoma. 2005 [cited 2008 Aug 15]; Available from: URL:http://www.emedicine.com

2. Raizada IN, Pratap VK, Mishra TD, Rohatgi VK. Orbital rhabdomyosarcoma. Indian J Ophthalmol. 1974;22:26-7.

3. Chaudhuri PM, Sen A, Sen SC, Gupta KPS, Chakravorthy S. Rhabdomyosarcoma. Indian J Ophthalmol. 1980;28(1):27-9.

4. Division of Pediatric Surgery, University of Miami Miller School of Medicine. Rhabdomyosarcoma: Surgical perspective.
[Online]. 2007 [Cited 2008 Oct 10]. Available from: URL:http:/ / emedicine.medscape.com/article/939156-overview

5. Department of Otolaryngology-Head and Neck Surgery, University of North Carolina School of Medicine. Rhabdomyosarcoma. [Online]. 2005 [Cited 2008 Oct 12]. Available from: http://emedicine.medscape.com/ article/873546-overview

6. Kapoor S, Sood GC, Aurora AL, Sood M. Alveolar Rhabdomyosarcoma. Indian J Ophthalmol. 1976;24(3):27-9. 